\title{
Preoperative response to corticosteroids predicts success of laparoscopic splenectomy in treating immune thrombocytopenia
}

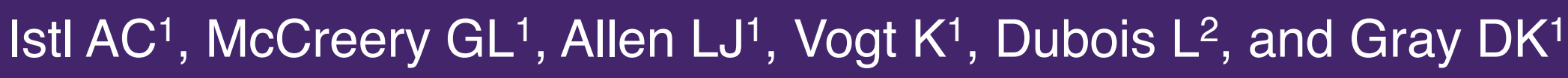

\section{BACKGROUND}

The optimal therapeutic sequence for ITP is controversial. The American Society of Hematology 2011 guidelines recommend splenectomy as second-line therapy in patients who do not respond to steroids (Grade 1B). ${ }^{1}$ Some practitioners defer surgical referral to avoid peri-operative morbidity and mortality despite a lack of evidence directly comparing non-operative interventions to splenectomy. ${ }^{2,3}$

Laparoscopic splenectomy has a lower adverse event rate than many medical therapies. ${ }^{4-6}$ Identifying factors that predict surgical success pre-operatively may change the second-line interventions being offered.

\section{ObJective}

Determine factors predictive of LS success which can guide therapeutic decision-making and inform prognosis.

\section{Methods}

A single-centre retrospective review of ITP patients who had LS at London Health Science Centre from 2000 to 2015 was conducted.

Platelet response to therapy and LS success were determined on a threshold of $50 \times 10^{9} /$ L. $^{16}$ Corticosteroid response was stratified: complete responders maintained platelets $>50 \times 10 \% / L$ after stopping steroid therapy. Transient responders achieved a platelet count $>50 \times 10^{9} / \mathrm{L}$ but relapsed on therapy. Non-responders did not produce platelet count $>50 \times 10^{9} / \mathrm{L}$. The first course of corticosteroids was assessed because 98\% of patients received first-line corticosteroids.

Univariate analysis assessed differences between LS failure and LS success groups. Multivariate logistic regression was for factors independently associated with success of LS. A subanalysis was completed comparing individuals $<65$ years to $\geq 65$ years with respect to complication rate and LS success.

\section{RESULTS}

141 patients had attempted LS. 3.6\% were converted to open. Successful disease remission was achieve in 111 patients (78.7\%). 30-day complication rate was $8.5 \%$. One patient developed bacteremia. $98 \%$ of patients had pre-operative steroid therapy: $50.7 \%$ had a complete response, $20.3 \%$ had a transient response, and $29 \%$ showed no response. Response to corticosteroids was associated with LS success ( $p<0.01)$ (Figure 1).

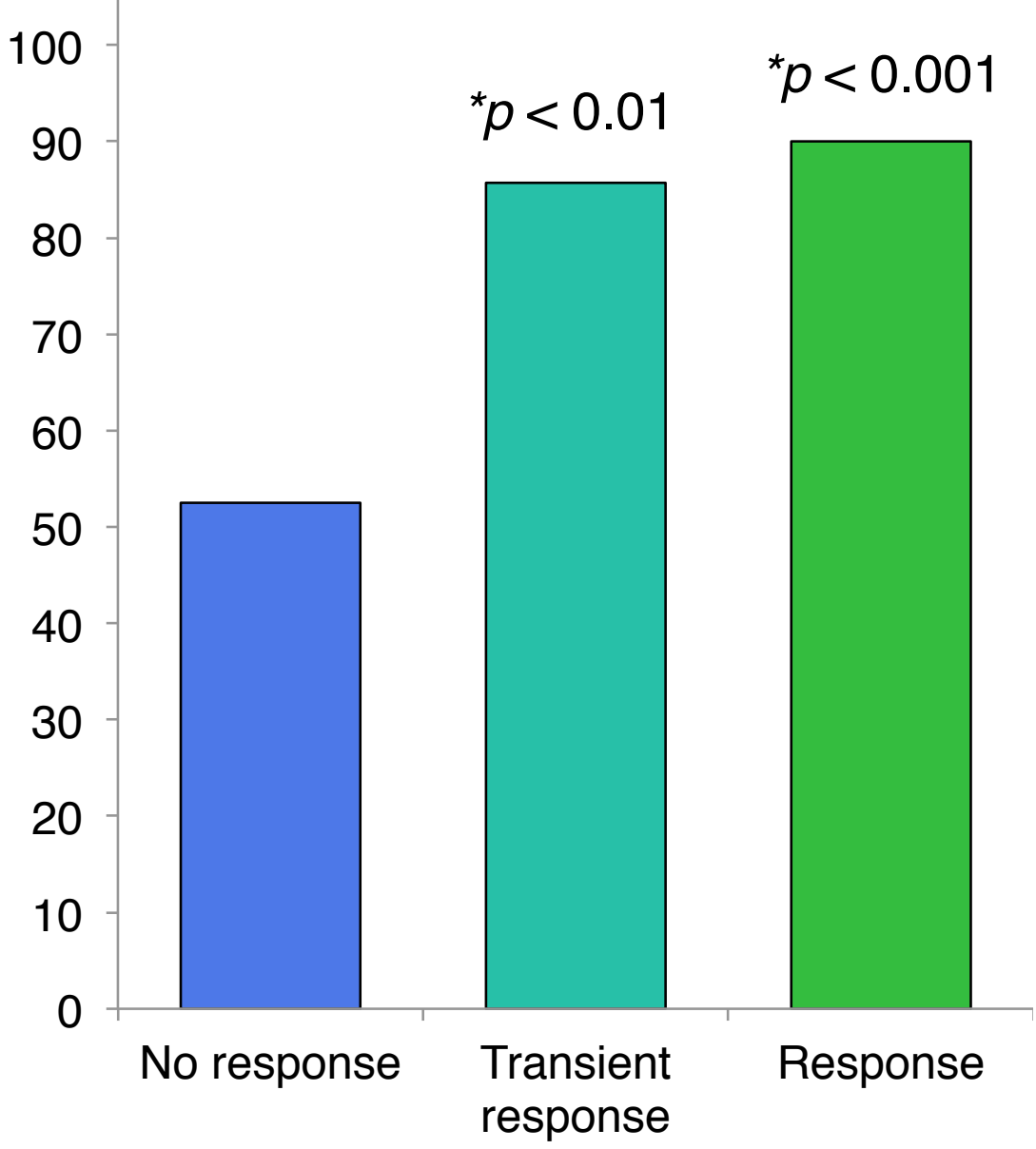

Response to initial course of steroids

Figure 1. Response to initial corticosteroid course and LS success.

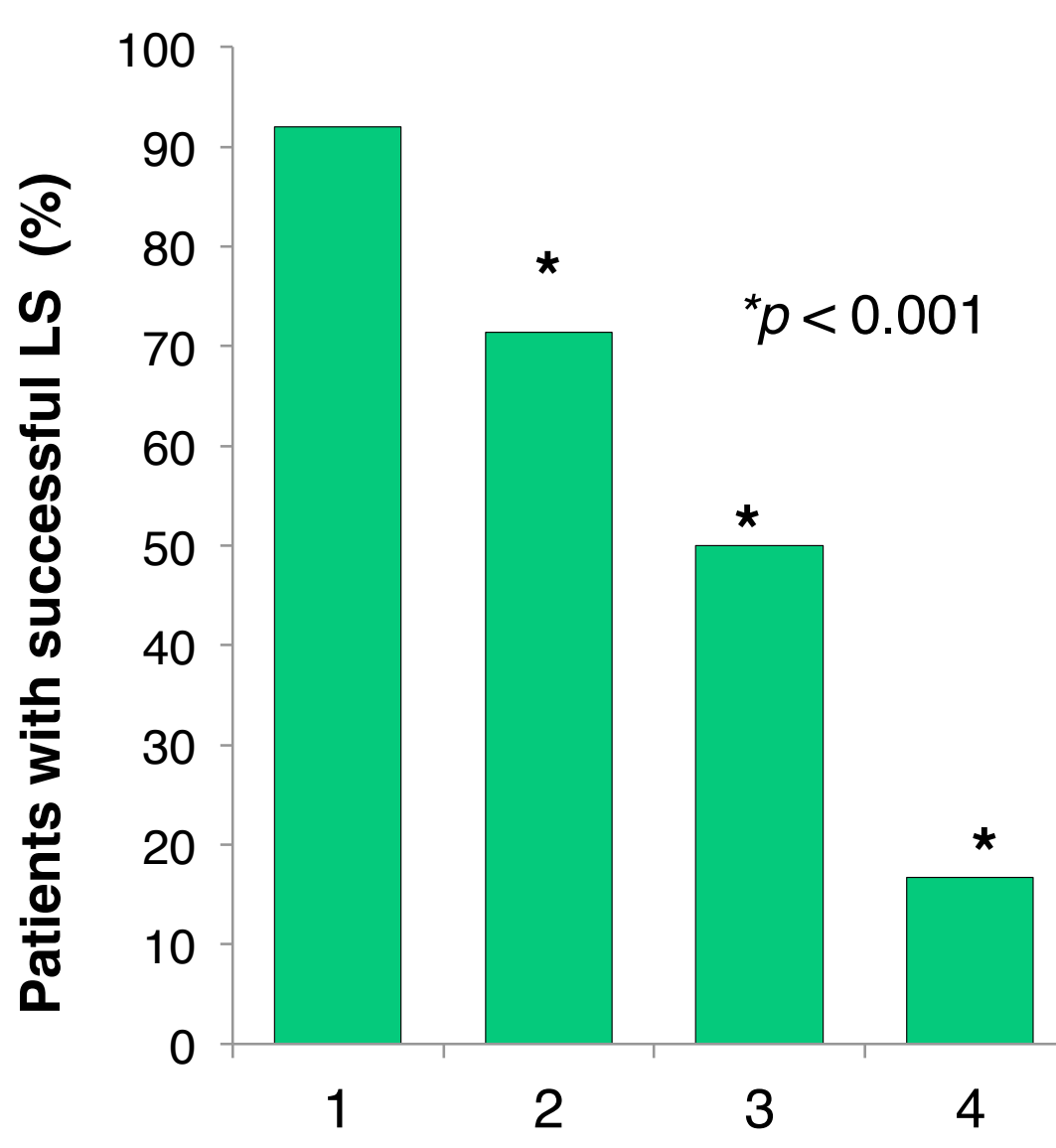

Number of Medical Interventions

Figure 2. Number of preoperative medical therapies and LS success.
Corticosteroid response predicted improved odds of LS success for transient and complete responders (Table 1). Each additional preoperative medical therapy reduced odds of LS success versus those receiving fewer interventions (Figure 2). Subanalysis of individuals $\geq 65$ showed no increased risk of LS failure $(p=0.27)$ or complications $(p=0.15)$.

Table 1. Multivariate logistic regression for factors independently predictive of LS success.

\begin{tabular}{|c|c|c|}
\hline & $p$ value & OR [95\%Cl] \\
\hline Age & 0.957 & $1.00[0.975-1.027]$ \\
\hline $\begin{array}{l}\text { Number of pre-operative medical } \\
\text { therapies }\end{array}$ & $<0.001$ & $0.27[0.136-0.544]$ \\
\hline $\begin{array}{c}\text { Response to corticosteroids } \\
\text { Transient } \\
\text { Complete }\end{array}$ & $\begin{array}{l}<0.05 \\
<0.01\end{array}$ & $\begin{array}{l}4.15[1.131-15.231] \\
5.58[1.870-16.665]\end{array}$ \\
\hline
\end{tabular}

\section{Conclusions}

1. LS is a safe, effective procedure for treating ITP and age should not preclude referral.

2. LS achieved disease remission in $90 \%$ of steroid-responsive patients and $78.7 \%$ of patients overall. Steroid response independently predicts LS success.

\section{REFERENCES}

\section{DISCLOSURES}

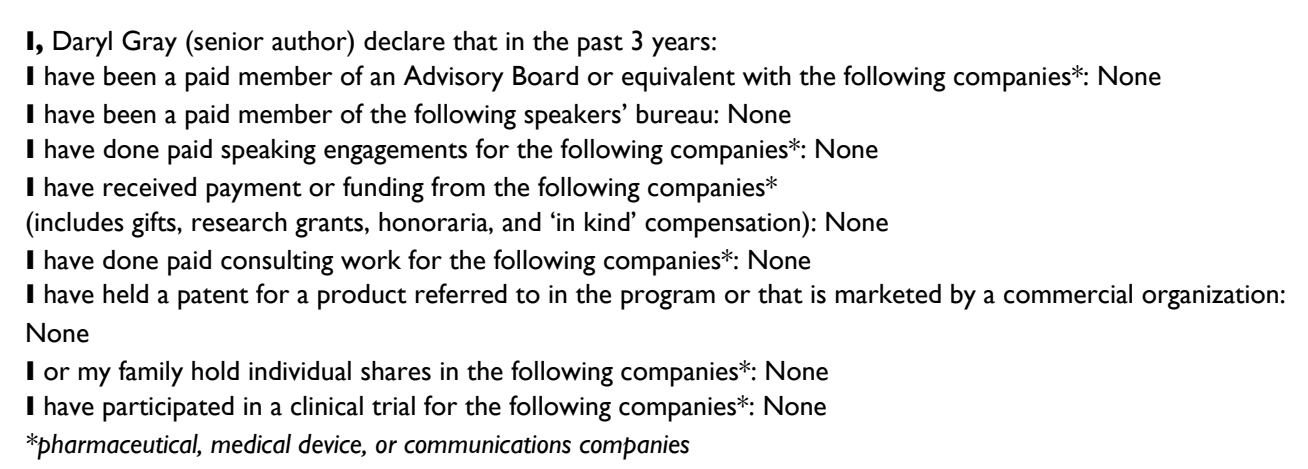

